\title{
Low-Power Chip-Scale Rubidium Plasma Light Source for Miniature Atomic Clocks
}

\author{
Vinu Venkatraman, Herbert Shea \\ Microsystems for Space Technologies Laboratory \\ École Polytechnique Fédérale de Lausanne (EPFL) \\ Neuchâtel, Switzerland \\ vinu.venkat@epfl.ch
}

\author{
Yves Petremand, Nico de Rooij \\ Sensors, Actuators and Microsystems Laboratory \\ École Polytechnique Fédérale de Lausanne (EPFL) \\ Neuchâtel, Switzerland
}

\author{
Christoph Affolderbach, Gaetano Mileti \\ Laboratoire Temps-Fréquence \\ University of Neuchâtel \\ Neuchâtel, Switzerland
}

\begin{abstract}
We present the development, testing and characterization of a low-power chip-scale Rubidium (Rb) plasma light source designed to serve for optical pumping in miniature atomic clocks. The technique used is electrodeless capacitively coupled plasma (CCP) discharge, driven in a microfabricated $R b$ vapor cell. The device is electrically driven at frequencies between 1 and $36 \mathrm{MHz}$ to emit $140 \mu \mathrm{W}$ of stable optical power while coupling $<6 \mathrm{~mW}$ of electrical power to the discharge cell. To our knowledge this is the first reported $\mathbf{R b}$ plasma emitted from a chip-scale device.
\end{abstract}

\section{INTRODUCTION}

Compact $\mathrm{Rb}$ atomic clocks (overall volumes of 100$1000 \mathrm{~cm}^{3}$ ), are widely used in many applications including navigation systems, network synchronizations and digital communications [1]. A common commercial implementation of such an atomic clock is the compact $\left(\sim 100 \mathrm{~cm}^{3}\right)$ Rubidium microwave frequency standard based on the DoubleResonance (DR) technique, using a glass-blown Rb plasma discharge lamp to achieve optical pumping. With the advent of microtechnology there has been an upsurge in portable devices (GPS receivers, for example) leading to a growing need for smaller, lower-cost, chip-scale atomic clocks with reduced power consumption, that show better frequency stability than the currently used quartz crystal oscillators. This has led to research towards miniaturizing atomic clocks to reach volumes of few $\mathrm{cm}^{3}$ or below $[2,3]$, mostly based on the Coherent Population Trapping (CPT) approach that has been preferred over the traditional DR technique [4].

The majority of $\mathrm{Rb}$ DR clocks use Rb plasma discharge lamps as the light source for optical pumping due to the lamp's intrinsically correct frequency ( $\mathrm{Rb}$ lines), technical simplicity, very slow frequency drift (aging), and long lifetime. However, because these glass-blown lamps usually consume several watts of electrical power and are not easily integrable with an ideal chip-stack configuration, chip-scale $\mathrm{Rb}$ DR clocks had been deemed a challenge and are relatively unexplored. The CPT technique was preferred for chip-scale clocks by using a laser diode (VCSEL) as the light source for atomic resonance $[2,3]$ because of their easy integration, good power efficiency, planarity and compact dimensions $\left(\sim \mathrm{mm}^{2}\right)$. However, the VCSEL approach requires critical temperature control, sophisticated control electronics for laser frequency stabilization, and complicated setup procedures [2].

The goal of this research is to develop a microfabricated planar low-power $\mathrm{Rb}$ plasma light source (as shown in operation in Fig. 1) to exploit the inherent advantages of a Rb discharge lamp, avoiding the issues faced by the VCSEL and providing an alternative and innovative design for chip-scale $\mathrm{Rb}$ atomic clocks.

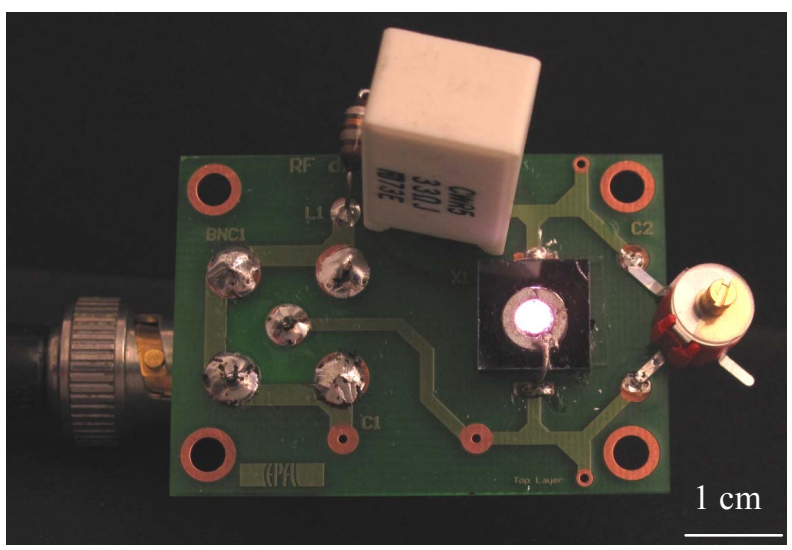

Figure 1. Photographic view of the $\mathrm{Rb}$ plasma light source emitting the $\mathrm{Rb}$ glow discharge

This project was funded by Swiss National Science Foundation (SNSF) Sinergia grant CRSI20_122693. 
Microfabrication of hermetically sealed $\mathrm{Rb}$ cells with volumes on the order of $1 \mathrm{~mm}^{3}$ has been a challenge, with several groups reporting different bonding techniques compatible with $\mathrm{Rb}$ filling, for instance anodic bonding $[3,5]$ or soldering $[6,7]$. Such cells have been used as the reference cell in miniaturized atomic frequency standards (atomic clocks), but not as light sources - in part due to the additional complexity of igniting a stable plasma. While first studies on micro-cell plasma light sources reported on low-power raregas discharges, no Rb light was reported $[8,9]$.

\section{A. Double Resonance Rb Atomic Clock}

The fundamental concept behind timekeeping in atomic clocks is to exploit an atomic resonance as reference for stabilizing the frequency of a quartz oscillator. In a $\mathrm{Rb}$ atomic clock [6], this resonance is the "clock" transition between the two ground-state hyperfine levels of ${ }^{87} \mathrm{Rb}$, at a microwave frequency near $6.83 \mathrm{GHz}\left(5^{2} \mathrm{~S}_{1 / 2}\right.$ state, $\left|\mathrm{F}=2, \mathrm{~m}_{\mathrm{F}}=0\right\rangle$ to $\mid \mathrm{F}=1$, $\left.\mathrm{m}_{\mathrm{F}}=0\right\rangle$ ), detected on atoms maintained in a small $\mathrm{Rb}$ reference cell. In a double-resonance Rb clock, pump light from a lamp or laser resonant with the Rb D1 or D2 line $(794.8 \mathrm{~nm}$ or $780.2 \mathrm{~nm}$, respectively) irradiates the cell and creates a ground-state polarization in the atoms. The clock transition is then detected by applying a microwave field to the atoms via a microwave-cavity placed around the cell, and the resonance manifests itself as a narrow dip in pump-light power transmitted through the cell. The frequency of a voltagecontrolled quartz oscillator is locked to this dip using phasesensitive detection.

Our envisaged double-resonance $\mathrm{Rb}$ atomic clock consists of a micro-fabricated $\mathrm{Rb}$ plasma light source, a microfabricated $\mathrm{Rb}^{85}$ filter, a micro-fabricated $\mathrm{Rb}$ reference cell which is enclosed by a miniaturized microwave cavity, and a photodetector (Fig. 2). The Rb plasma light source intrinsically emits at the correct wavelengths, Rb D1 and D2 lines, as required in a $\mathrm{Rb}$ clock.

\section{B. Rb Plasma Glow Discharge}

Sustaining a plasma glow discharge in a $\mathrm{Rb}$ cell requires continuous ionization of $\mathrm{Rb}$ vapor atoms. A sufficiently strong electric field, when applied across a $\mathrm{Rb}$ vapor cell, can ionize the $\mathrm{Rb}$ vapor atoms to generate $\mathrm{Rb}$ ions and free electrons.

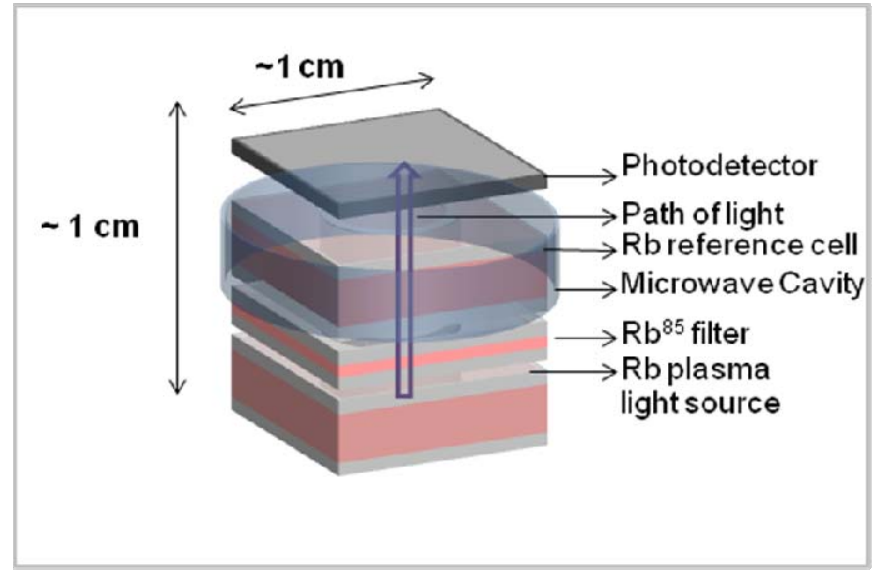

Figure 2. Schematic diagram of the envisioned Rb DR chip-scale atomic clock with the integrated $\mathrm{Rb}$ light source
By maintaining a sufficiently strong electric field, the electrons and ions are accelerated towards the electrodes, and many of them collide with more neutral vapor atoms to generate positive ions and electrons through impact ionization before they reach the electrodes. This becomes an avalanche process, eventually initiating an electrical breakdown leading to several possible types of gas discharges [10], including the $\mathrm{Rb}$ plasma glow discharge emitted as radiation by $\mathrm{Rb}$ atoms during their radioactive decay from the excited state to the ground state.

The $\mathrm{Rb}$ vapor density is very low at room temperature $\left(\sim 10^{-6}\right.$ millibars of vapor pressure at $\left.30^{\circ} \mathrm{C}\right)$, hence requiring an extremely high field strength to ionize the $\mathrm{Rb}$ atoms (Paschen curve) [11]. In order to reduce the field and hence the electrical power to ignite the glow discharge, an inert lowpressure starter gas is added to the cell. The temperature of the cell is also increased to up to $150^{\circ} \mathrm{C}$ by using a resistive heater, in order to increase the $\mathrm{Rb}$ vapor density in the cell. As $\mathrm{Rb}$ is highly reactive with many gases, a few ten millibars of an inert gas are used as starter gas - in our case Ar, which has a relatively smaller diameter and high mean free path among noble gases with an ionization potential of $15.75 \mathrm{eV}$ [10].

\section{DEVICE MiCROFABRICATION AND DEVELOPMENT}

\section{A. Design and Microfabrication}

The design approach chosen for the Rb plasma light source is the electrodeless capacitively coupled plasma (CCP), sustained in a planar micro-fabricated $\mathrm{Rb}$ cell. Capacitive coupling produces stronger field lines in a planar cell cavity and therefore makes it easier to ignite a plasma than the more widely used inductive coupling which is more efficient for spherical cells.

The micro-fabricated $\mathrm{Rb}$ cell (outer dimensions of $1 \mathrm{x} 1 \mathrm{x}$ $\left.0.3 \mathrm{~cm}^{3}\right)$, consists of a stack of three layers: Pyrex $(500 \mu \mathrm{m}$ thick), Silicon $(2 \mathrm{~mm})$, Pyrex $(500 \mu \mathrm{m})$ which enclose a $\Phi=$ $5 \mathrm{~mm}, h=2 \mathrm{~mm}$ cylindrical cavity in which few microliters of Rubidium and 70 millibars of Argon are hermetically sealed by a two-step anodic bonding process (Fig. 3). First, the cavity is created by DRIE etching a through-hole in the silicon wafer. Then, the etched silicon wafer is anodically bonded to the bottom Pyrex layer.

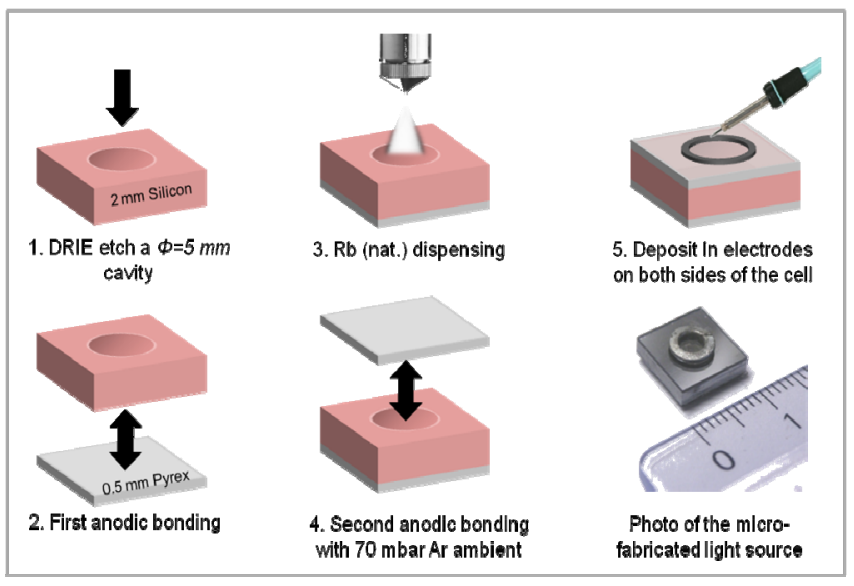

Figure 3. Schematic diagram showing the Rb light source microfabrication process flow 
After this first bonding step, the natural $\mathrm{Rb}$ is dispensed into the cavity under vacuum by an atomic jet, 70 millibars of Argon are introduced in the chamber, and the top Pyrex layer is anodically bonded to the bottom layers to create the hermetically sealed Rb mini-cell [12].

A $200 \mu \mathrm{m}$ thick $100 \%$ Indium solder ring or film is deposited on top of both sides of the Pyrex layers (i.e., outside the cell) to act as capacitive parallel electrodes to capacitively couple RF power to the cell. 100\% Indium wets glass well and is hence a good choice for deposition on the Pyrex walls. The $100 \%$ Indium electrodes are deposited by placing and aligning Indium pre-form rings on top of Pyrex layers above the cavity and reflowing using a soldering iron. These external electrodes avoid electrode corrosion and might allow for lower power consumption when compared to the more traditional internal electrode designs [13]. Note that in a future waferlevel production of the plasma light source, the electrodes can be directly integrated onto the Pyrex layers used to close the cell by evaporation or sputtering and micropatterning techniques, and could be made of a suitable metal other than Indium in order to better withstand high temperatures. Our micro discharge cell design is thus compatible with wafer level manufacturing of many devices at a time, potentially allowing for greatly reduced costs per unit.

\section{B. RF Capacitively Coupled Plasma (CCP) Circuit}

The technique used to power the light source is RF electrodeless CCP. The microfabricated $\mathrm{Rb}$ vapor cell has an intrinsic capacitance of $\sim 0.4 \mathrm{pF}$ at low frequencies $(<10 \mathrm{MHz})$ and up to $\sim 1.6 \mathrm{pF}$ at higher frequencies $(<36 \mathrm{MHz})$, and a reactance of $\sim \mathrm{j} 85 \mathrm{k} \Omega$ at low frequencies (few $\mathrm{MHz}$ ).

The output impedance of the RF generator used as signal source is $(50+\mathrm{j} \cdot 0 \Omega)$ hence requiring careful impedance matching of the load to equal the source to $(50+\mathrm{j} \cdot 0 \Omega)$ using L-C components. This enables high power and voltage amplification across the cell to achieve the required breakdown voltage with the lowest input electrical power.

Fig. 4 shows schematically the RF plasma ignition circuit connected to the impedance-matched load constituting the light source. The two main components in the impedance matching network are (1) a capacitor in parallel to the cell to

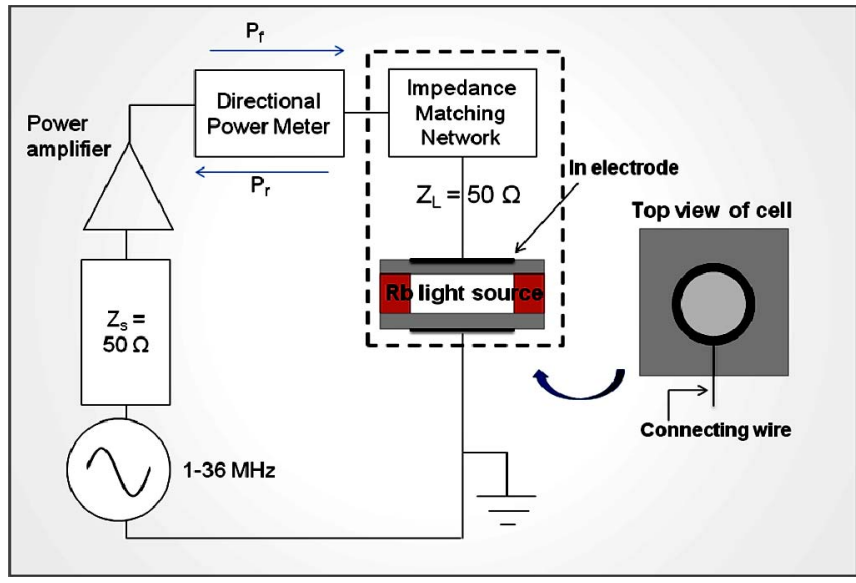

Figure 4. Schematic circuit of the RF electronic circuitry and impedance matching for plasma ignition reduce the overall capacitive reactance and (2) an inductor in series with the above capacitors to cancel out the reactive load to form a purely resistive load. The values and the Equivalent Series Resistance (ESR) of the components are carefully chosen to maximize the power transfer to the cell.

\section{DEVICE CHARACTERIZATION}

The light source was impedance matched and tested at several frequencies over the $1 \mathrm{MHz}$ to $36 \mathrm{MHz}$ frequency range. The results reported in this article were obtained at 4.6 $\mathrm{MHz}$.

\section{A. Output optical characteristics}

The output optical spectrum of the light source was measured using a spectrometer and is shown in Fig. 5. At cell temperatures above $\sim 90^{\circ} \mathrm{C}$ strong Rb D1 lines (794.8 nm) and D2 lines $(780.2 \mathrm{~nm})$ are observed. These lines rapidly rise in intensity with increasing cell temperature, as expected with the rapidly rising $\mathrm{Rb}$ vapor pressure inside the cell.

The total optical power of the light source was $140 \mu \mathrm{W}$ when $6 \mathrm{~mW}$ of electrical power was coupled to the cell. The total optical power emitted on the Rb D2 line was $15 \mu \mathrm{W}$, at a cell temperature of $150^{\circ} \mathrm{C}$.

\section{B. Rb light power stability}

The stability of the output optical power of the $\mathrm{Rb}$ lines was recorded over time for the plasma light source operating at a temperature of $100^{\circ} \mathrm{C}$. The short-term (seconds) and long term (hours) variation in optical power was measured.

The average short-term fluctuations in optical intensity of the $\mathrm{Rb}$ lines was found to be less than $0.1 \%$ and the long-term variations were found to be less than 5\% (shown in Fig. 6). The primary cause of the long-term variations in optical power is the drift in resistance $(2-3 \%)$ of the electrical components of the impedance matching network with time, mainly due to thermal effects. These long-term variations can be limited to less than $0.01 \%$ using a PID feedback loop that controls the input electrical power and/or the RF drive frequency to maintain a steady output light power level.

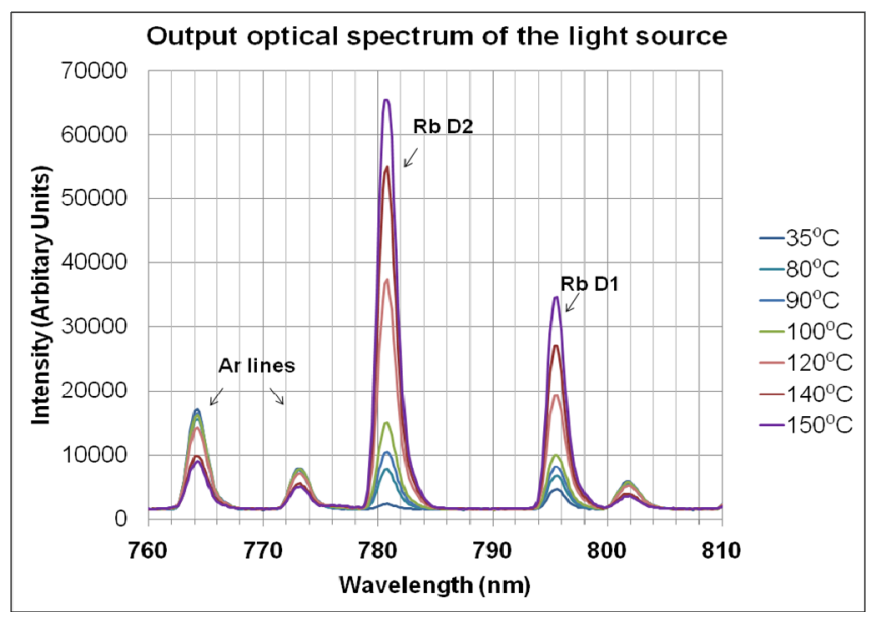

Figure 5. Optical spectrum of the light source at different temperatures between $\left(35-150^{\circ} \mathrm{C}\right)$. The spectral width of the lines is limited by the resolution of the spectrometer used here. 


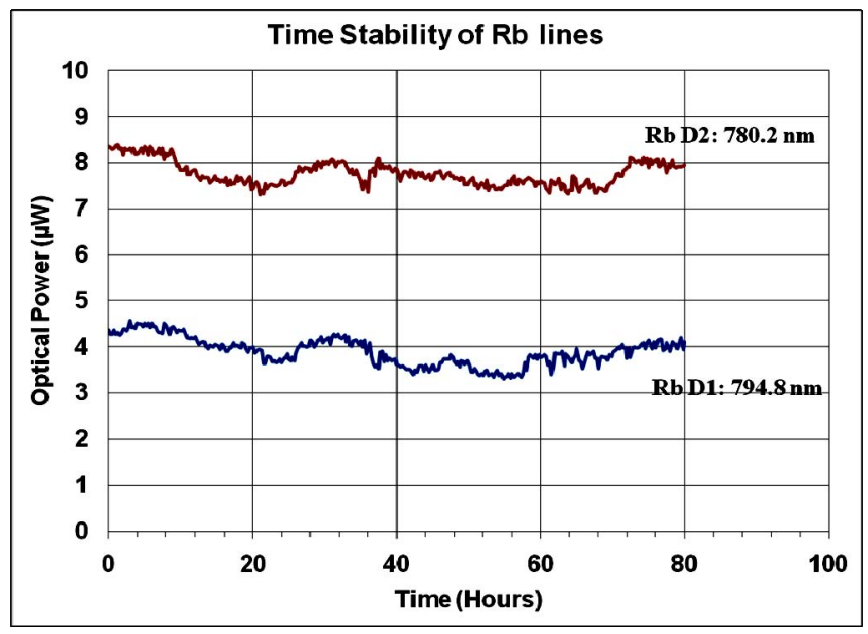

Figure 6. Stability of the $\mathrm{Rb}$ lines with time, measured at $100{ }^{\circ} \mathrm{C}$

\section{SUMMARY AND FUTURE WORK}

A stable and low-power chip-scale Rb plasma light source with potential for wafer-scale fabrication has been demonstrated, intended for use in chip-scale atomic clocks. The light source emits several $\mu \mathrm{W}$ of optical power at the 780.2 and $794.8 \mathrm{~nm} \mathrm{Rb}$ lines from a $0.3 \mathrm{~cm}^{3}$ chip stack. A successful $\mathrm{Rb}$ hermetic sealing technique through standard microfabrication processes has been demonstrated that withstands the plasma operation. The external electrode design ensures the longevity of the device by avoiding electrode erosion by the plasma.

When combined with a micro-fabricated ${ }^{85} \mathrm{Rb}$ filter cell [9], the presented plasma light source can be used for hyperfine optical pumping of $\mathrm{Rb}$ in a novel miniature atomic clock.

Testing of this light source as an optical pump in a test DR atomic clock setup to prove its functionality is planned. In the current circuit design, the efficiency of power coupled to the cell is low, thus current research is geared towards increasing this efficiency. Different starter gases and pressures are to be explored to find the best performance conditions.

\section{ACKNOWLEDGMENT}

We thank our project group members for their support. We acknowledge the SNSF R'Equip program for the funding of the High speed data acquisition test bench used in this study.

\section{REFERENCES}

[1] J. Camparo, "The rubidium atomic clock and basic research", Phys. Today, vol. 60, pp. 33-39, 2007

[2] S. Knappe, "MEMS atomic clocks", Comprehensive Microsystems, vol. 3, pp. 571-612, 2007.

[3] A. Douahi, L. Nieradko, J. C. Beugnot, J. Dziuban, H. Maillote, S. Guerandel, M. Moraja, C. Gorecki and V. Giordano : "Vapour microcell for chip scale atomic frequency standard", Electronics Letters, vol. 43, pp. 279-280, 2007.

[4] A. M. Braun, et al., "RF-interrogated end-state chip-scale atomic clock", Proc. $39^{\text {th }}$ Annual Precise Time and Time Interval Meeting (PTTI), USA, pp.233 - 247, 2007.
[5] S. Knappe, V. Shah, P. Schwindt, L. Holberg, J. Kitching, L. Liew and J. Moreland, "A microfabricated atomic clock", Appl. Phys. Lett., vol. 85, pp. 1460-1462, 2004.

[6] F. Vecchio, V. Venkatraman, H. Shea, T. Maedar and P. Ryser, "Dispensing and hermetic sealing $\mathrm{Rb}$ in a miniature reference cell", Sensors \& Actuators: A. Physical, in press.

[7] Y. Pétremand, C. Schori, R. Straessle, G. Mileti, N. de Rooij and P. Thomann, "Low temperature indium-based sealing of microfabricated alkalicells for chip scale atomic clocks", Proc. of the European Frequency and Time Forum (EFTF), Noordwijk, the Netherlands, p.119, 2010 .

[8] H. Guo and A. Lal, "Self-powered photon source", IEEE Proc. of the 12th Int. Conference on Solid State Sensors, Actuators and Microsystems, Transducers 2003, U.S.A, pp. 1474-1477, 2003.

[9] S. Wang, L. Lin and H. Guo, "Analysis and design of a micromachined $\mathrm{Rb}-85$ filter in passive rubidium atomic clock", Proc. IEEE Int. Conference on Nano/Micro Engineered and Molecular Systems, China, pp. 911-914, 2009.

[10] E. Nasser, "Fundamentals of gaseous ionization and plasma electronics", Wiley-Interscience, 1971.

[11] F. Paschen, "Über die zum funkenübergang in luft, wasserstoff und kohlensäure bei verschiedenen drücken erforderliche potentialdifferenz", Weid. Annalen der Physik, vol.37, pp. 69-75, 1889.

[12] J. DiFrancesco, F. Gruet, C. Schori, C. Affolderbach, R. Matthey, G. Mileti, Y. Salvadé, Y. Petremand and N. De Rooij, "Evaluation of the frequency stability of a VCSEL locked to a micro-fabricated rubidium vapour cell", Proc. SPIE, vol. 7720, pp. 77201T, 2010.

[13] D. O. Wharmby, "Electrodeless lamps for lighting: a review", IEEProceedings, vol. 140, pp. 465-473, 1993. 